\title{
A SEARCH FOR ISOTOPIC ANOMALIES IN URANIUM
}

\section{J. H. Chen and G. J. Wasserburg}

The Lunatic Asylum, Division of Geological and Planetary Sciences, California Institute of Technology, Pasadena, California 91125

Abstract. The ${ }^{238} \mathrm{U} /{ }^{235} \mathrm{U}$ ratios for nine bulk chondritic meteorites and a terrestrial basalt were measured. The total range in ${ }^{238} \mathrm{U} /{ }^{235} \mathrm{U}$ determined for both total meteorites and for acid leaches was from 137.2 to 138.3 and to within the error limits indistinguishable from normal terrestrial U. The typical errors in a single determination are $\pm 6 \%\left(2 \sigma_{\mathrm{M}}\right)$ for a $2 \mathrm{ng} U$ sample from a chondrite. Taking the extreme values of $\delta^{235} \mathrm{U}$ for each measurement the maximum amount of excess ${ }^{235} \mathrm{U}$ that can be allowed to be present ranges from $2 \times 10^{8}$ to $2 \times 10^{9}$ atoms per gram of bulk meteorite. These results do not support the claims of variations in ${ }^{238} \mathrm{U} /{ }^{235} \mathrm{U}$ at the percentage levels or number of excess ${ }^{235} \mathrm{U}$ atoms in some of the same meteorites as reported by several other previous workers.

Introduction. The purpose of this investigation is to measure the relative abundances of ${ }^{238} \mathrm{U}$ and ${ }^{235} \mathrm{U}$ in various classes of chondrites. Until recently isotopic variations were not expected and only limited high precision work has been done in search of them. In addition, as ${ }^{235} \mathrm{U}$ is relatively rare in meteoritic material, there has been very little effort directed toward determining the ${ }^{238} \mathrm{U} /{ }^{235} \mathrm{U}$ ratio in these materials with high precision and reliability. Numerous measurements on terrestrial materials show no large variations except in special circumstances [Naudet and Renson, 1975]. The "absolute" ratio of terrestrial $U$ is $\left({ }^{238} \mathrm{U} /{ }^{235} \mathrm{U}\right)_{\oplus}=137.88$ [Shields, 1960; Cowan and Adler, 1976], possibly being uncertain to $\pm 1 \%$. Measurements on lunar materials show a ${ }^{238} \mathrm{U} /{ }^{235} \mathrm{U}$ ratio indistinguishable from normal $\mathrm{U}$ [Rosholt and Tatsumoto, 1971; Fields et al., 1972; Barnes et al., 1972] . Arden [1977] reported analyses of five chondrites which showed that some samples contained an excess of ${ }^{235} \mathrm{U}$. In particular, excesses of ${ }^{235} \mathrm{U}(290 \%)$ were reported for some bulk chondrites and certain fractions of dissolution residues gave excesses up to $2400 \%$. The results reported by Arden were astounding both in terms of the magnitude of the isotopic shifts and the number of excess nuclei required. These effects would require $4 \times 10^{9}$ to $7 \times 10^{10}$ excess ${ }^{235} \mathrm{U}$ atoms per gram of bulk chondrite and would jmply a high yield ( $x 40$ to 300 ) at $Z=92$ for the injected material compared to the value of $1 \sigma^{3} \cdot 1 \sigma^{4}$ estimated for intermediate $\mathrm{Z}$ assuming solar abundances. [See Wasserburg, Papanastassiou and Lee, 1979]. Arden's report stimulated work on U isotopes in meteorites. Chen and Tilton [1979] and Unruh, Hutchison and Tatsumoto [1979a,b] did not observe large effects, but did report

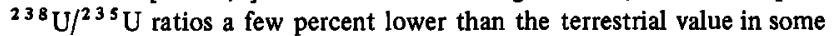
chondrites and Allende inclusions. However, Tatsumoto and Shimamura [1979] reported ${ }^{238} \mathrm{U} /{ }^{235} \mathrm{U}$ ratios from 128 to 145 for several meteorites.

To clarify this matter, we carried out analyses on nine meteorites and a terrestrial rock. Prior to carrying out the measurements, we considered that the major technical problems should first be addressed. Our approach was (1) to improve the ionization efficiency of U; (2) to firmiy establish the limits of detection and reliability by measurement of enriched standards; and (3) to eliminate sources of contamination from "exotic" $U$ isotopes of anthropogenic origin.

Experimental Procedures. Because of our concern over contamination, our first step was to clean the laboratory area, establish cleaner chemical procedures, use only new chemical processing equipment, and to stop any handling of ${ }^{235} \mathrm{U}$ during the period of the work. The usual procedure for measuring $U$ is by isotope dilution using pure ${ }^{235} \mathrm{U}$ as a tracer and results in the presence of pure ${ }^{235} \mathrm{U}$ in the laboratory. The contamination levels necessary to produce a one percent shift in ${ }^{235} \mathrm{U}$ abundance for a gram of chondrite are $2 \times 10^{9}$ atoms of ${ }^{235} \mathrm{U}$ (equivalent to a particle of $2000 \AA$ ). The possibility of contamination from such sources must be a cause of great concern since the vogue of isotopic variations provokes a kind of isotopic credulity which inhibits some of us from critically

Copyright 1980 by the American Geophysical Union. examining our measurements. An example of anthropogenic contamination was reported by Piepgras, Wasserburg, and Dasch [1979] for ${ }^{148} \mathrm{Nd}$ at the level of $10^{6}$ atoms/gm of seawater due to the unrecognized presence of this isotope in the laboratory from previous work.

$A^{233} \mathrm{U}^{236} \mathrm{U}$ double spike prepared from high purity ${ }^{233} \mathrm{U}$ and ${ }^{236} \mathrm{U}$ was used to monitor mass dependent isotope fractionation. The absolute ratio of ${ }^{233} \mathrm{U} /{ }^{236} \mathrm{U}$ and the concentration in the mixed spike were determined using a solution of mixed spike and the NBS U-500 standard $\left[99.94 \% \mathrm{U}_{3} \mathrm{O}_{8},{ }^{235} \mathrm{U} /{ }^{238} \mathrm{U}=0.9997 \pm 0.001\right.$, Garner, Machlan, and Shields, 1971] to correct for fractionation. The ratio in the double spike, ${ }^{233} \mathrm{U} /{ }^{236} \mathrm{U}=1.0066$ is known to within $\pm 1 \%$ excluding the errors of $1 \%$ reported for NBS U-500. Following Arden [1977], we used graphite as an emitter. The procedure used by us was to load a suspension of graphite in water onto a previously outgassed Re filament. This was evaporated yielding a thin uniform layer of graphite. The $U$ samples in $0.1 \mathrm{~N} \mathrm{HNO}_{3}$ were then loaded on the filament and dried. Another load of graphite suspension was then deposited and dried making a sandwich with $U$ between the graphite layers. This technique routinely yields $\sim 1 \%$ ionization efficiency and low loading blank $(<0.5 \mathrm{pg})$. The ion beam intensity $\left(\mathrm{U}^{+}\right)$for $2 \mathrm{ng} \mathrm{U}$ was $5 \times 10^{-13} \mathrm{~A}$ between $1700^{\circ}-1750^{\circ} \mathrm{C}$ for $2-3 \mathrm{hrs}$. At each mass the zeroes and peaks were measured with an integration time of $1 \mathrm{sec}$ and a settling time of $3 \mathrm{sec}$ prior to signal integration. To determine instrumental mass fractionation, we measured the ion beams (I) at masses 233 and 236 in conjunction with 235 and 238 . The mass jumping sequences were cycled in the order ${ }^{238} \mathrm{U}^{236} \mathrm{U}^{233} \mathrm{U}^{238} \mathrm{U}$ and ${ }^{236} \mathrm{U}^{235} \mathrm{U}^{235} \mathrm{U}^{233} \mathrm{U}^{236} \mathrm{U}$ and data taken in sets of 10 ratios. The ratios (relative to ${ }^{236} \mathrm{U}$ ) in a set were averaged and corrected for discrimination $(<2 \% / \mathrm{mu})$ and contributions of ${ }^{238} \mathrm{U}$ and ${ }^{235} \mathrm{U}$ from the double spike which were less than $4 \times 10^{-2} \%$ and $7 \%$ respectively. In general, three sets of 10 ratios $\left({ }^{238} \mathrm{U}:{ }^{236} \mathrm{U}:{ }^{233} \mathrm{U}\right)$ were first measured, the filament current then raised such that $I_{236}$ was similar to that of $I_{23}$ in the preceding sets and three sets of ${ }^{236} \mathrm{U}:{ }^{235} \mathrm{U:}{ }^{233} \mathrm{U}$ were measured. The ${ }^{238} \mathrm{U} /{ }^{235} \mathrm{U}$ ratio was calculated from the normalized ${ }^{238} \mathrm{U} /{ }^{236} \mathrm{U}$ and ${ }^{235} \mathrm{U} /{ }^{236} \mathrm{U}$ ratios in the different sets and the error given as the vector sum of the errors of the latter two ratios in $2 \sigma_{\mathrm{M}}$ (mean). To test linearity of the detection system the ${ }^{238} \mathrm{U} /{ }^{235} \mathrm{U}$ ratio was determined as a function of ion beam intensity. On the Lunatic III mass-spectrometer system we observed a decrease in the measured ${ }^{238} \mathrm{U} /{ }^{236} \mathrm{U}$ ratios with increasing signal. All experiments showed the same behavior which was probably caused by saturation of the detector. The error from this apparent deficiency in

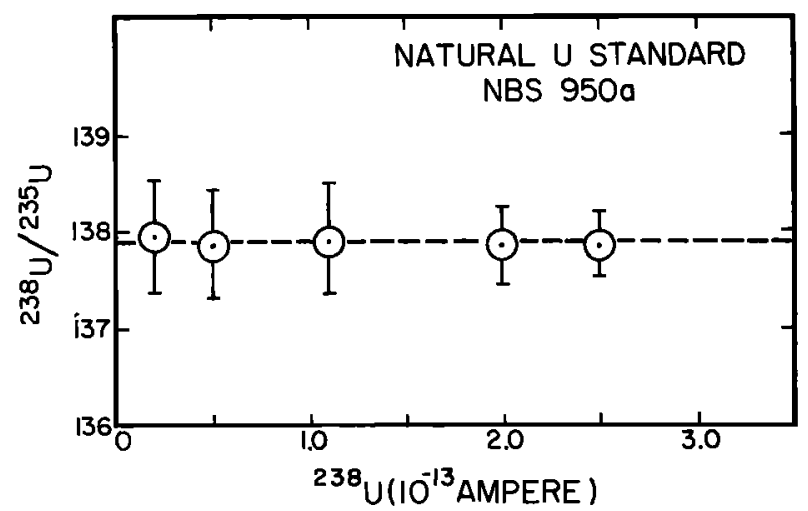

Fig. 1. Natural U standard, linearity test of the electron multiplier (current integration mode) on the Lunatic I mass spectrometer. The ${ }^{238} \mathrm{U} /{ }^{235} \mathrm{U}$ ratios are corrected for fractionation using the double spike. The normalized ${ }^{238} \mathrm{U} /{ }^{235} \mathrm{U}$ ratios measured with intensities of ${ }^{238} \mathrm{U}$ between 0.2 and $2.5 \times 10^{-13} \mathrm{~A}$ agree with the normal value. 


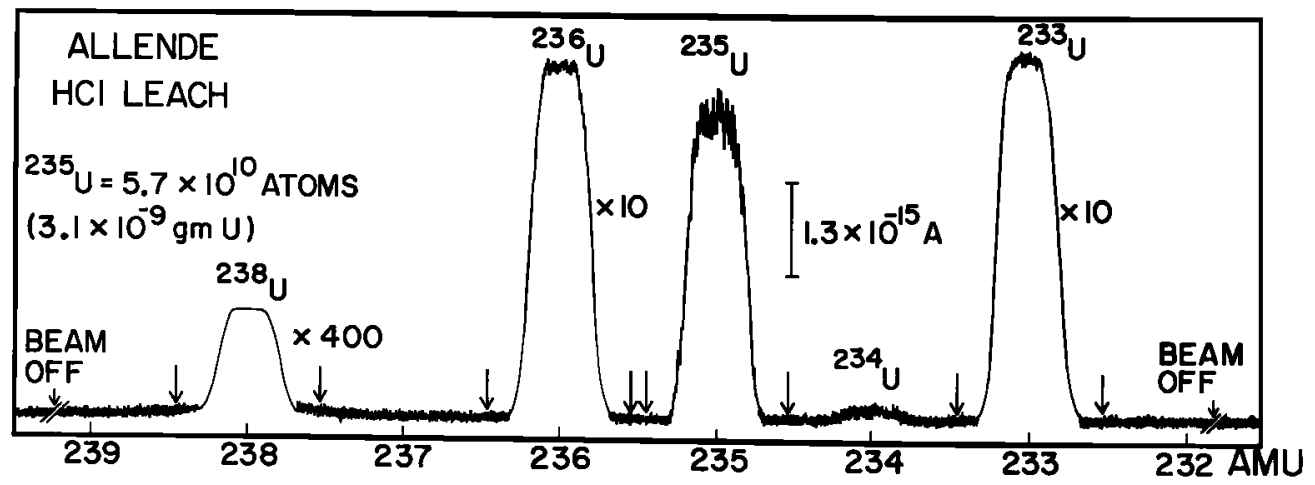

Fig. 2. U mass spectrum of $\mathrm{HCl}$ leach on Allende. Arrows show where the zeroes were measured. Note the shift in scale.

${ }^{238} \mathrm{U}$ would correspond to $\sim 35 \%$ enrichment of ${ }^{235} \mathrm{U}$ when $\mathrm{I}_{238}$ was $8 \times 1 \sigma^{-13}$ A. For comparison we performed the same experiments on the Lunatic I equipped with the same type of multiplier (gain $=4 \times 10^{3}$ at 2300 volts.) The results (Fig. 1) indicate that the problem of saturation is absent on the Lunatic I within limits of error. All experiments reported here were performed on the Lunatic I which was previously never used for $U$ measurements. During all experiments, we scanned the mass range 230-240 (see Fig. 2) and did not observe any signals besides U. To establish the reliability with which shifts in isotopic abundance can be determined, we prepared four standards enriched in ${ }^{235} \mathrm{U}$ by mixing different amounts of ${ }^{235} \mathrm{U}$ spike into a standard solution of NBS 950a and ${ }^{236} \mathrm{U}^{2}{ }^{33} \mathrm{U}$ tracer. The isotopic abundance of $U$ in the enriched $U$ standards was measured by loading different amounts of $U$ for each set of experiments. A complete set of comparisons was made for samples of enriched standards ranging from 2 to $20 \mathrm{ng}$. The measured ratios after correction are compared with gravimetrically determined ${ }^{238} \mathrm{U} /{ }^{235} \mathrm{U}$ ratios in Fig. 3. These results were indistinguishable for all the data and indicate that (1) at least $5 \% \mathrm{U}$ isotopic effects can be resolved for samples as small as $2 \mathrm{ng}$, and (2) all mass spectrometric measurements for the enriched standards agree with the values calculated from gravimetry well within errors $\left(2 \sigma_{M}\right)$.

Samples of meteorite fragments were prepared by removing all exterior surfaces of the samples as they were received in the laboratory. Samples (1-1.5 g) were digested in HF in a $50 \mathrm{ml}$ FEP teflon tube followed by $\mathrm{HNO}_{3}$ and $\mathrm{HClO}_{4}$ attack in a $30 \mathrm{ml} \mathrm{FEP}$ teflon beaker until all residues dissolved in $2 \mathrm{M} \mathrm{HCl}$. $U$ was coprecipitated with other group II major elements with $\mathrm{NH}_{4} \mathrm{OH}$ and separated by passing through Dowex AGl x 8, 100-200 mesh anion exchange resin using 7M $\mathrm{HNO}_{3}$ and $\mathrm{H}_{2} \mathrm{O}$. A second anion exchange resin was used to purify $U$ from Th using $6 \mathrm{M}$

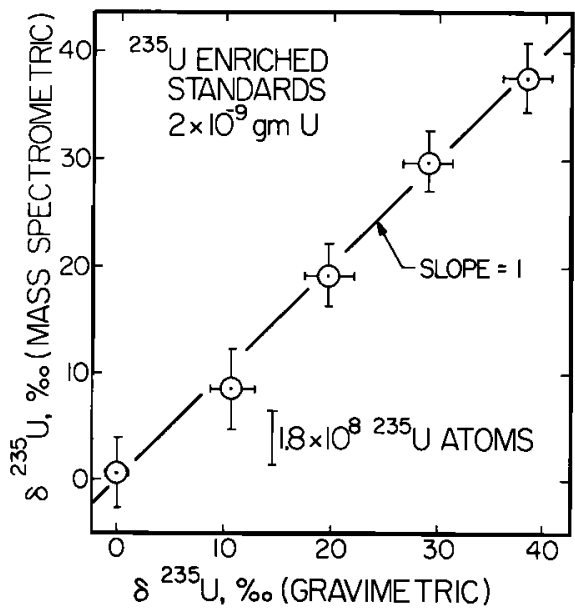

Fig. 3. Comparison between gravimetrically determined $\delta^{235} \mathrm{U}$ and mass spectrometrically measured $\delta^{235} \mathrm{U}$ for experiments on $2 \mathrm{ng} \mathrm{U}$ samples. All data plot on a $45^{\circ}$ line and demonstrate the ability to clearly resolve $\mathrm{U}$ isotopic effects above $5 \%$ for $2 \mathrm{ng} \mathrm{U}$.
$\mathrm{HCl}, 7 \mathrm{M} \mathrm{HNO}_{3}$, and $\mathrm{H}_{2} \mathrm{O}$. The chemical yield of $\mathrm{U}$ ranged from 50 to $95 \%$. The total procedural blank was $\sim 2 \times 10^{10}$ atoms $(\sim 8 \mathrm{pg})$. Acid leach experiments were done on two meteorites in the order listed. An aliquot $(\sim 10 \%)$ was spiked to determine the amount of $U$ and the balance then spiked to give ${ }^{238} U /{ }^{236} U \approx 12$. Each set of meteorite measurements was preceded by a linearity test as shown in Fig. 1. Results are shown in Table 1 listed chronologically.

\section{RESULTS AND DISCUSSION}

Results are given in Table 1 as ${ }^{238} \mathrm{U} /{ }^{235} \mathrm{U}$ and as fractional deviations $\left(\delta^{235} \mathrm{U}\right)$ of the ratios from the nominal $\left({ }^{235} \mathrm{U} /{ }^{238} \mathrm{U}\right)_{\oplus}$ value in parts in $10^{3}$. The amount of meteoritic $\mathrm{U}$ measured per analysis ranges from 0.2 to $10 \mathrm{ng}$. The results on terrestrial samples NBS 950a and basalt BCR-1 show good agreement with the assumed terrestrial value for samples of $U$ ranging from $1.7 \times 10^{-9}$ to $2 \times 10^{-8} \mathrm{gm}$. Two aliquots of $U$ from Dhajala, containing 1.8 and $4.4 \mathrm{ng} \mathrm{U}$ were run and are in good agreement with each other and the value is indistinguishable from terrestrial. These analyses followed an analysis of NBS 950a under the same range of operating conditions. A similar sequence of experiments were done on all the meteorite samples, which include a fragment of Richardton (originally from ASU) transferred to this laboratory from UCSB for analysis. The only apparent difference between the meteorties and the standards lies in the fact that the precision of the meteorite data was not usually of the quality obtained on the very pure standard.
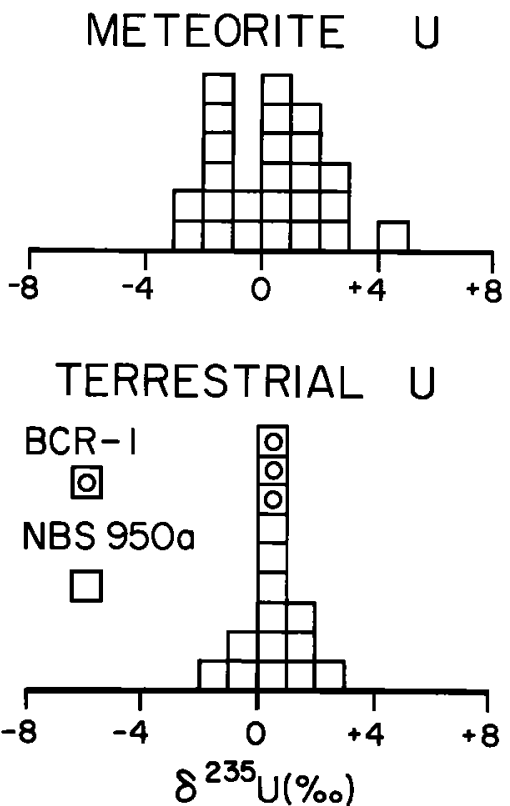

Fig. 4. Histograms showing the measured $\delta^{235} U$ on meteorite and terrestrial $U$. The distributions of both classes of data show normal distribution with means close to $\delta^{235} \mathrm{U}=0$, and a small dispersion. 
Table 1. U Isotopic Compositions ${ }^{\mathrm{a}}$

\begin{tabular}{|c|c|c|c|c|}
\hline Samples $^{b}$ & & $\mathrm{U}$ (ng) & ${ }^{238} U /{ }^{235} U$ & $\delta^{235} \mathrm{U}(\%)$ \\
\hline N1 NBS 950a & & 20 & $137.85 \pm 0.41$ & $+0.22 \pm 2.99$ \\
\hline N2 NBS 950a & & 20 & $137.86 \pm 0.33$ & $+0.15 \pm 2.39$ \\
\hline N3 NBS 950a & & 5 & $137.49 \pm 0.54$ & $+2.83 \pm 3.90$ \\
\hline N4 NBS 950a & & 2 & $137.77 \pm 0.44$ & $+0.80 \pm 3.19$ \\
\hline N5 NBS 950a & & 5 & $137.90 \pm 0.40$ & $-0.14 \pm 2.89$ \\
\hline \multirow[t]{3}{*}{ BCR-1 } & 1 & 20 & $137.83 \pm 0.37$ & $+0.36 \pm 2.69$ \\
\hline & 2 & 11 & $137.84 \pm 0.41$ & $+0.29 \pm 3.00$ \\
\hline & 3 & 1.7 & $137.83 \pm 0.83$ & $+0.36 \pm 5.97$ \\
\hline N6 NBS 950a & & 5 & $137.86 \pm 0.43$ & $+0.14 \pm 3.09$ \\
\hline \multirow[t]{2}{*}{ Dhajala (H3) } & 1 & 1.8 & $137.72 \pm 0.73$ & $+1.16 \pm 5.28$ \\
\hline & 2 & 4.4 & $138.12 \pm 0.55$ & $-1.74 \pm 3.98$ \\
\hline \multirow[t]{2}{*}{ Pultusk (H5) } & 1 & 1.6 & $137.58 \pm 0.99$ & $+2.18 \pm 7.16$ \\
\hline & 2 & 3.3 & $137.69 \pm 1.29$ & $1.38 \pm 9.33$ \\
\hline N7 NBS 950a & & 10 & $137.68 \pm 0.55$ & $+1.45 \pm 3.99$ \\
\hline \multirow[t]{2}{*}{ Richardton (H5) } & 1 & 2.7 & $138.14 \pm 0.65$ & $-1.88 \pm 4.67$ \\
\hline & 2 & 6.3 & $137.20 \pm 1.12$ & $+4.96 \pm 8.17$ \\
\hline \multirow{2}{*}{ Bruderheim (L6) } & 1 & 2.0 & $137.50 \pm 0.50$ & $+2.76 \pm 3.60$ \\
\hline & 2 & 9.2 & $138.18 \pm 1.10$ & $-2.17 \pm 7.92$ \\
\hline N8 NBS 950a & & 10 & $137.83 \pm 0.51$ & $+0.36 \pm 3.69$ \\
\hline \multirow[t]{2}{*}{ Allende (CV3) } & 1 & 5.1 & $138.28 \pm 0.69$ & $-2.89 \pm 4.96$ \\
\hline & 2 & 8.8 & $137.87 \pm 0.69$ & $+0.07 \pm 4.98$ \\
\hline N9 NBS 950a & & 10 & $137.67 \pm 0.48$ & $+1.53 \pm 3.49$ \\
\hline \multirow[t]{2}{*}{ Murchison (CM2) } & 1 & 1.0 & $138.09 \pm 1.39$ & $-1.57 \pm 9.90$ \\
\hline & 2 & 2.6 & $137.50 \pm 1.10$ & $+2.76 \pm 7.96$ \\
\hline N10 NBS 950a & & 10 & $137.63 \pm 0.29$ & $-1.82 \pm 2.10$ \\
\hline \multirow[t]{2}{*}{ St. Severin (LL6) } & 1 & 4.5 & $138.02 \pm 0.69$ & $-1.00 \pm 4.97$ \\
\hline & 2 & 5.7 & $137.98 \pm 0.74$ & $-0.72 \pm 5.37$ \\
\hline \multirow[t]{2}{*}{ Leedey (L6) } & 1 & 2.8 & $138.03 \pm 0.72$ & $-1.08 \pm 5.17$ \\
\hline & 2 & 5.4 & $137.81 \pm 0.51$ & $+0.51 \pm 3.69$ \\
\hline \multirow{4}{*}{ Richardton (H5) } & & 10 & $137.85 \pm 0.40$ & $+0.22 \pm 2.89$ \\
\hline & $\mathrm{HF}+\mathrm{HCl}^{\mathrm{c}}$ & 1.9 & $137.62 \pm 0.50$ & $+1.86 \pm 3.60$ \\
\hline & $\mathrm{HNO}_{3}$ & 5.4 & $137.76 \pm 0.47$ & $+0.88 \pm 3.39$ \\
\hline & $\mathrm{HClO}_{4}$ & 1.2 & $138.09 \pm 1.38$ & $-1.52 \pm 9.88$ \\
\hline N12 NBS 950a & & 10 & $137.88 \pm 0.41$ & $0.00 \pm 2.99$ \\
\hline \multirow[t]{4}{*}{ Allende (CV3) } & $\mathbf{H C l}^{\mathrm{d}}$ & 3.1 & $137.80 \pm 0.49$ & $+0.58 \pm 3.59$ \\
\hline & $\mathrm{HNO}_{3}$ & 2.6 & $137.65 \pm 1.13$ & $+1.67 \pm 8.15$ \\
\hline & HF & 5.6 & $137.88 \pm 0.41$ & $0.00 \pm 3.00$ \\
\hline & $\mathrm{HClO}_{4}$ & 4.5 & $137.75 \pm 0.83$ & $+0.94 \pm 5.97$ \\
\hline N13 NBS 950a & & 10 & $138.03 \pm 0.28$ & $-1.08 \pm 2.09$ \\
\hline \multirow[t]{2}{*}{ Richardton (H5) } & 1 & 2.2 & $137.69 \pm 0.44$ & $+1.38 \pm 3.19$ \\
\hline & 2 & 10.4 & $138.07 \pm 0.33$ & $-1.36 \pm 2.3$ \\
\hline \multirow{3}{*}{$\begin{array}{l}\text { N14 NBS 950a } \\
\text { Barwell (L5) }\end{array}$} & & 18 & $137.85 \pm 0.26$ & $+0.22 \pm 1.89$ \\
\hline & 1 & 0. & $137.58 \pm 1.24$ & $+2.18 \pm 8.94$ \\
\hline & 2 & 5.1 & $137.51 \pm 0.39$ & $+2.70 \pm 2.81$ \\
\hline
\end{tabular}

a Errors are $2 \sigma_{\mathrm{M}}$. All data corrected for mass fractionation and a small contribution of ${ }^{235} \mathrm{U}$ from the ${ }^{233} \mathrm{U}^{236} \mathrm{U}$ double spike.

$$
\delta^{235} \mathrm{U}=\left(\left({ }^{235} \mathrm{U} /{ }^{238} \mathrm{U}\right)_{\text {meas }} \times 137.88-1\right) \times 10^{3}
$$

bSamples no. 14 from G. R. Tilton; Richardton (nos. 3, 9 and 11), \#100h, Arizona State University; Murchison (no. 6), \#Me 2642, Field Museum of Natural History, Chicago; St. Severin (no. 7), \#H2402-b, Museum of Natural History, Paris; Leedey (no. 8), \#489.1, Arizona State University. Numbers after sample name denote repeat runs on aliquots of same solution. "Sample $(1.48 \mathrm{gm})$ decomposed in HF. Dried residue heated in $6 \mathrm{M} \mathrm{HCl}(10 \mathrm{ml})$, and clear solution centrifuged giving entry labelled $\mathrm{HF}+\mathrm{HCl}$. The residue was dried again, and $7 \mathrm{M} \mathrm{HNO} \mathrm{HN}_{3}(10 \mathrm{ml})$ added and heated. Solution removed and remainder brought into total solution with $70 \% \mathrm{HClO}_{4}(2 \mathrm{ml}) .{ }^{d}$ Sample $(1.75 \mathrm{gm})$ leached in $6 \mathrm{M} \mathrm{HCl}$ $(20 \mathrm{ml})$ for five days. The solution centrifuged and the residue washed twice with $\mathrm{H}_{2} \mathrm{O}$. The washes were combined with the $\mathrm{HCl}$ extract and labelled $\mathrm{HCl}$. The residue was leached with a new acid $(15 \mathrm{ml})$ in each successive cycle. After $\mathrm{HClO}_{4}(2 \mathrm{ml})$ leach, no residue was observed.
On the basis of the enriched $U$ standard experiments, we are confident that we can resolve any $\mathrm{U}$ isotopic effect above $5 \%$. The results show that the ${ }^{238} \mathrm{U} /{ }^{235} \mathrm{U}$ ratios for the individual meteorites studied are similar within error limits to that for normal terrestrial $U$. The average of all meteoritic $U$ isotopic measurements is ${ }^{238} \mathrm{U} /{ }^{235} \mathrm{U}=137.84 \pm 0.52$ $\left(2 \sigma_{\mathrm{GM}}\right)$ or $\delta^{235} \mathrm{U}=+0.3 \pm 3.8 \%$, which is indistinguishable from the average for the $U$ standard, $137.79 \pm 0.28\left(2 \sigma_{\mathrm{GM}}\right)$ or $\delta^{235} \mathrm{U}=$ $+0.7 \pm 2.1 \%$. Here the errors are $2 \sigma$ of the grand means of each class of samples. To illustrate better the distribution of these results, the $\delta^{235} \mathrm{U}$ values are shown in histograms in Fig. 4. Most of the meteoritic U measurements have uncertainties about $6 \%$ (see a bar graph in Fig. 5). Taking the extreme $\delta^{235} \mathrm{U}$ value of +13.13 for bulk Richardton (Aliquot 2), the amount of excess ${ }^{235} \mathrm{U}$ per gram which can be present is $2 \times 10^{9}$ atoms. Similar calculations for the rest of the data indicate that the maximum amount of excess ${ }^{235} \mathrm{U}$ per gram which can be allowed within the errors ranges from $2 \times 10^{8}$ to $10^{9}$ atoms. In contrast, the results of Arden [1977] indicate a ${ }^{235} \mathrm{U}$ excess of from $4 \times 10^{9}$ to $7 \times 10^{10}$ atoms per gram for some meteorites. We did not observe the shifts in ${ }^{238} \mathrm{U} /{ }^{235} \mathrm{U}$ in some meteorites reported by Chen and Tilton [1979], Unnuh et al., [1979a,b], and Tatsumoto and Shimamura [1979]. A direct comparison with the results of Chen and Tilton was possible. The solution aliquots of all these meteorites on which $U$ isotopes had been measured were transferred from UC Santa Barbara to this laboratory and measured. These solutions yielded ${ }^{238} \mathrm{U} /{ }^{235} \mathrm{U}$ ratios ranging from 133.8 to 137.3 , confirming each of the values of Chen and Tilton. These more precise results clearly showed $\delta^{235} \mathrm{U}$ ranging from 30.5 to 4.2. Through the courtesy of G. R. Tilton, fragments of the original meteorites (Dhajala, Pultusk, and Bruderheim), which showed excess ${ }^{235} \mathrm{U}$ in the solutions prepared at UCSB, were transferred to CIT and processed as described here. The results (Table 1, nos. 1, 2 and 4), clearly demonstrate a normal value. We conclude that the abundance measurements reported by Chen and Tilton were correct but that the effects are spurious and probably due to laboratory contamination with ${ }^{235} \mathrm{U}$. From our observations we conclude that ${ }^{238} \mathrm{U} /{ }^{235} \mathrm{U}$ in the bulk meteorites studied is similar to the terrestiral value to well within an extreme error of $\pm 5 \%$ and considering the dispersion about the mean probably to within an error of $\pm 2 \%$. The uncertainty in the ${ }^{238} \mathrm{U} / /^{235} \mathrm{U}$

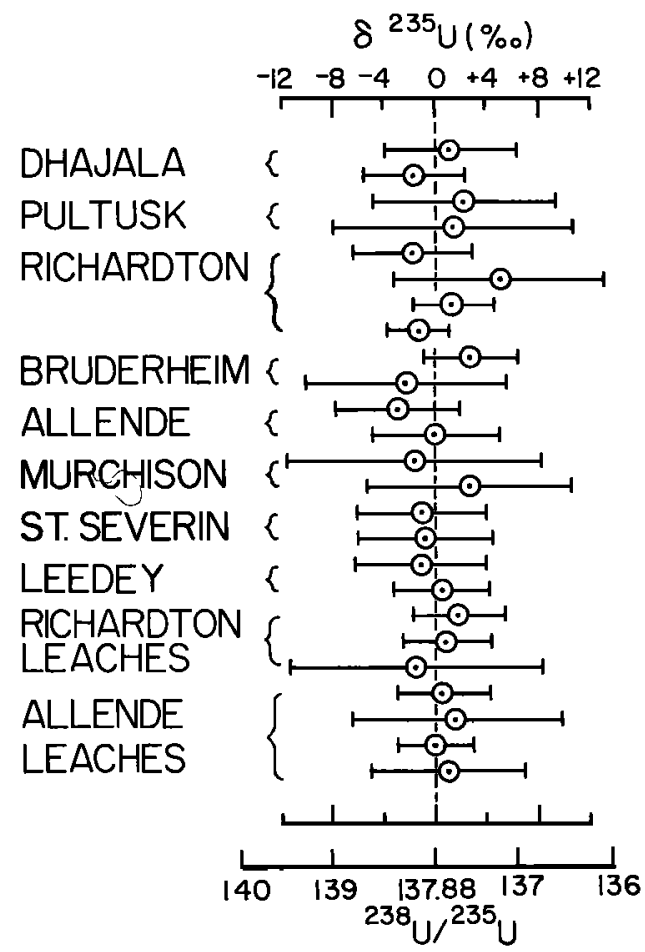

Fig. 5. Bar graph showing the range of $\delta^{235} \mathrm{U}$ on bulk meteorites and leaches. The center dashed line corresponds to the nominal terrestrial value of $\left({ }^{238} \mathrm{U}^{235}\right)_{\oplus}=137.88$. 
ratio in this study constrains the amount of any excess ${ }^{235} \mathrm{U}$ to less than $2 \times 10^{9}-2 \times 10^{8}$ atoms per gram of bulk meteorite.

Leaching experiments were carried out on whole rock samples of Richardton and Allende. The Richardton sample was from the same parent sample analyzed by Arden [1977]. The leaching procedure generally followed the same sequence of acid treatments used by Arden [1977], but the conditions were different and the fraction of total $U$ leached in each step differed from the yields obtained by him. The results obtained on Richardton show that two successive leaches and the dissolution of the final residue contained 22,64 , and $14 \%$ of the $U$ and that the isotopic compositions were all the same as the terrestrial value to within error. A modified leaching procedure was used for Allende with yields of $20,16,35$, and 29 percent, respectively, and again giving the terrestrial value. We find no evidence for the existence of isotopically peculiar $U$ in either bulk samples or leaches using the procedures followed here. From data reported by Arden on Richardton (no. 2), it would appear that $57 \%$ of the total $\mathrm{U}$ is isotopically anomalous with $\delta^{235} \mathrm{U}=500 \%$. If only $10 \%$ of this exotic $U$ were present in one of our Richardton leaches, we would have observed a shift of $\delta^{235} \mathrm{U}=$ $45.200 \%$ which was not observed. Similarly, $43 \%$ of the total $U$ in Arden's Allende (no. 2) appear to have $\delta^{235} U=42 \%$. If $10 \%$ of this anomalous $U$ were present in our Allende leaches, we would have observed a shift of $\delta^{235} \mathrm{U} \sim 12 \%$ which was also not observed. In addition we analyzed another larger sample of Richardton (\#11) to obtain high precision data. Aliquots of $U$ from this sample are indistinguishable from normal $U$ to within $1.4 \%$. Our repeated analyses of different fragments of Richardton thus show no hint of a $U$ anomaly. After submission of this paper we also analyzed a sample of Barwell chondrite for which Unruh et al., [1979] reported $238 / 235 \mathrm{U}$ ratio of $135.24 \pm 0.17$. The results $(\# 12)$ show that the ${ }^{238} \mathrm{U} /{ }^{235} \mathrm{U}$ ratio for the bulk Barwell are similar within errors to terrestrial. We can find no evidence in support of isotopically anomalous $U$ in either bulk meteorite samples or in any of the leaches on two meteorites in which anomalies have been reported.

In previous studies of $\mathrm{Pb}-\mathrm{U}$ ages one of the limiting experimental errors lies in determining the number of ${ }^{238} \mathrm{U}$ atoms in a sample (typically errors of $\sim 1-1.5 \%$ ). This error is an order of magnitude larger than errors in the ratio of ${ }^{207} \mathrm{~Pb} /{ }^{206} \mathrm{~Pb}$. As a result, uncertainties in age determinations may be governed by errors in the ${ }^{206} \mathrm{~Pb} /{ }^{238} \mathrm{U}$ ratio. This is most serious in the dating of lunar samples where the data often form a linear array nearly tangent to the concordia curve [cf. Tera and Wasserburg, 1974]. In the experiments reported here, the ratio of ${ }^{238} \mathrm{U}$ to ${ }^{236} \mathrm{U}$ (or ${ }^{233} \mathrm{U}$ ) has always been better than $1.2 \%\left(2 \sigma_{\mathrm{M}}\right)$. It follows that the use of a mixed ${ }^{233} \mathrm{U}-{ }^{236} \mathrm{U}$ spike calibrated against a good standard can readily permit ${ }^{238} \mathrm{U}$ determinations at a precision of at least $1 \%$ on samples down to $1 \sigma^{10} \mathrm{gm}$ of $U$.

\section{CONCLUSIONS}

We find that the mean ${ }^{238} \mathrm{U} /{ }^{235} \mathrm{U}$ ratios for nine bulk meteorites to is indistinguishable from normal terrestrial uranium to within $4 \%$. The maximum amount of excess ${ }^{235} \mathrm{U}$ which can be allowed for these samples within the extreme limits of errors ranges from $2 \times 10^{8}$ to $2 \times 10^{9}$ atoms per gram of meteorite. Analysis of different leach fractions of two meteorites shows the isotopic compositions to be indistinguishable from normal terrestrial $U$. The upper limit to excesses of ${ }^{235} \mathrm{U}$ in all of the leach fractions is $3-9 \times 10^{8}$ atoms out of a total of $\sim 10^{11}$ atoms. From these observations we conclude that bulk chondrites have a $U$ isotopic composition which is the same as terrestrial to within limits of error $(2-4 \%)$. We further conclude that there is no evidence in support of variations in the ${ }^{238} \mathrm{U} /{ }^{235} \mathrm{U}$ abundances at the percentage levels or in terms of the excess ${ }^{235} \mathrm{U}$ atoms as reported by Arden [1977] or in support of the small shifts in ${ }^{238} \mathrm{U} /{ }^{235} \mathrm{U}$ reported by Chen and Tilton [1979], Unruh et al. [1979a,b], and Tatsumoto and Shimamura [1979]. It is our belief that some of the effects may be due to contamination in the laboratory with exotic uranium of anthropogenic origin. Some small isotopic shifts may also be the result of instrumental errors. The scientific significance of variations in ${ }^{238} \mathrm{U} /{ }^{235} \mathrm{U}$ is sufficiently great that further and more intensive investigations must be pursued. Insofar as we have not repeated the specific acid decomposition procedures used by Arden
[1977], it is still possible that effects may exist in phases which have not been studied in this laboratory. However, unless the most extreme care is made to eliminate laboratory contamination and instrumental bias, it will be difficult to avoid the appearance of spurious shifts in isotopic abundances in nature. The level of isotopic shifts which are anticipated in bulk samples may be estimated. If we assume that the deficiency in ${ }^{135} \mathrm{Ba}$ of $\sim 2$ parts in $10^{4}$ or the ${ }^{129} \mathrm{I} /{ }^{127} \mathrm{I}$ ratio of $\sim 10^{-4}$ is a measure of the number of exotic nuclei added to the solar system and that the chemical abundance of the injected material is roughly similar to solar, then we would expect shifts of $\delta^{235} \mathrm{U} \sim 0.2 \%$ in bulk meteorites.

This study supports one of the basic assumptions used in U-Pb chronology of meteorites regarding the near constancy of ${ }^{238} U /{ }^{235} U$. The improved analytical techniques described here have direct application to the precise measurement of $U$ concentrations and the reliable determination of $\mathrm{U} \cdot \mathrm{Pb}$ ages for lunar and meteorite samples.

Acknowledgements. We have benefited from extensive discussion both philosophical and scientific with D. A. Papanastassiou. We are grateful to G. R. Tilton for generously giving us meteorite samples and aliquots of meteoritic U solutions. This work was supported by NSF grant PHY76-83685 and NASA grant NGL 05-002-188. Contribution 3351 (334) of the Division of Geological and Planetary Sciences.

\section{REFERENCES}

Arden, J. W., Isotopic composition of uranium in chondritic meteorites, Nature, 269, 788-789, 1977.

Barnes, I. L., B. S. Carpenter, E. L. Garner, J. W. Gramlich, E. C. Kuehner, L. A. Machlan, E. J. Maienthal, J. R. Moody, L. J. Moore, T. J. Murphy, P. J. Paulsen, K. M. Sappenfield, and W. R. Shields, Isotopic abundance ratios and concentrations of selected elements in Apollo 14 samples, Proc. Third Lunar Sci. Conf., 1465-1472, 1972.

Chen, J.H., and G. R. Tilton, Uranium Isotopic Composition in Chondritic Meteorites, Lunar and Planetary sci. X, 192-194, 1979.

Cowan, G. A., and H. H. Adler, The variability of the natural abundance of ${ }^{235} \mathrm{U}$, Geochim. Cosmochim. Acta, 40, 1487-1490, 1976.

Fields, P. R., H. Diamond, D. N. Metta, D. J. Rokop, and C. M. Stevens, ${ }^{23} \mathrm{~Np},{ }^{236} \mathrm{U}$, and other actinides on the moon, Proc. Third Lunar Sci. Conf., 1637-1644, 1972

Garner, E. L., L. A. Machlan, and W. R. Shields, Standard Reference Materials: Uranium Isotopic Standard Reference Materials, National Bureau of Standards Special Publication, 260-27, 1971.

Naudet, R., and C. Renson, Résultats des analyses systématiques de teneurs isotopiques de l'uranium, Proc. Int. Symp. Oklo Phenomenon, Libreville, 265-291, 1975.

Piepgras, D. J., G. J. Wasserburg, and E. J. Dasch, The isotopic composition of $\mathrm{Nd}$ in different ocean masses, Earth Planet. Sci. Lett., 45, 223-236, 1979.

Rosholt, J. N., and M. Tatsumoto, Isotopic composition of thorium and uranium in Apollo 12 samples, Proc. Second Lunar Sci. Conf., 1577.1584, 1971.

Shields, W. R., Comparison of Belgian Congo and Synthetic "Normal" samples, Report No. 8, U.S. National Bureau of Standards Meeting of the Advisory Committee, May 17 and 18, 37 pp., 1960.

Tatsumoto, M., and T. Shimamura, Isotopic composition of uranium in Allende and other chondrites, Meteoritics, 14,543-544, 1979.

Tera, F., and G. J. Wasserburg, U-Th-Pb systematics on lunar rocks and inferences about lunar evolution and the age of the moon, Proc. Fifth Lunar Sci. Conf., 1571-1599, 1974.

Unruh, D. M., R. Hutchison, and M. Tatsumoto, U-Th-Pb systematics and uranium isotopic composition of chondrites, Lunar and Planetary Sci. $X, 1256-1258,1979$ a.

Unruh, D. M., R. Hutchison, and M. Tatsumoto, U-Th-Pb age of the Barwell chondrite: Anatomy of a "discordant" meteorite, Proc. Tenth Lunar Sci. Conf., 1011-1030, 1979 b.

Wasserburg, G. J., D. A. Papanastassiou, and T. Lee, Isotopic heterogeneities in the solar system, Varenna Vol., Italian Physical Soc., 1979.

(Received January 4, 1980; accepted January 28, 1980.) 